QUARTERLY OF APPLIED MATHEMATICS

VOLUME LXIX, NUMBER 3

SEPTEMBER 2011, PAGES 549-568

S 0033-569X(2011)01249-7

Article electronically published on May 9, 2011

\title{
THE ONE-DIMENSIONAL FLOW OF A FLUID WITH LIMITED STRAIN-RATE
}

\author{
BY \\ A. FARINA (Università degli Studi di Firenze, Dipartimento di Matematica "U. Dini", Viale \\ Morgagni 67/a, 50134 Firenze, Italy), \\ A. FASANO (Università degli Studi di Firenze, Dipartimento di Matematica "U. Dini", Viale \\ Morgagni 67/a, 50134 Firenze, Italy), \\ L. FUSI (Università degli Studi di Firenze, Dipartimento di Matematica "U. Dini", Viale Morgagni \\ 67/a, 50134 Firenze, Italy), \\ AND \\ K. R. RAJAGOPAL (Department of Mechanical Engineering, Texas A\&M University, College \\ Station, Texas 77845)

\begin{abstract}
We present a model for a continuum in which the strain rate depends
\end{abstract} \\ linearly on the stress, as long as the latter is below a fixed threshold, but it is frozen \\ to a constant value when the stress exceeds such a threshold. The constitutive equation \\ is given in an implicit form as the stress is a multi-valued function of the strain rate. \\ We derive the model in a general 3D setting and we study the one-dimensional case of a \\ pressure-driven flow between two parallel plates. We prove some analytical results and \\ describe a procedure to determine the main physical parameters (stress threshold and \\ viscosity) by means of a rotational viscometer. Finally we show that the model can be \\ obtained as the limit case of a piecewise linear viscous model.
}

1. Introduction. This paper concerns the response of implicit fluid models, which are quite different from the ones commonly employed. In the case of the classical linearly viscous (Newtonian/Navier-Stokes) fluid, the generalized Stokesian fluid 1 or a general

Received March 24, 2010.

2010 Mathematics Subject Classification. Primary 76A05, 74D10, 35R35, 35K10.

Key words and phrases. Non-Newtonian fluids, implicit constitutive relations, free boundary problems, parabolic equations.

E-mail address: farina@math.unifi.it

E-mail address: fasano@math.unifi.it

E-mail address: fusi@math.unifi.it

E-mail address: krajagopal@mengr-tamu.org

${ }^{1} \mathrm{~A}$ fluid is referred to as a generalized Stokesian fluid if the Cauchy stress in the fluid is a function of the symmetric part of the velocity gradient and the density of the fluid.

(C) 2011 Brown University 
simple fluid 2 one prescribes an expression for the Cauchy stress in terms of kinematical quantities such as the symmetric part of the velocity gradient, the history of the deformation gradient, etc. However, not all fluids can be reasonably modeled within such a framework. Striking examples are the extremely shear-thickening colloidal suspensions [25. Within such a framework, one cannot obtain a fluid model wherein the fluid is incompressible but the material moduli of the fluid are functions of the pressure (Lagrange multiplier that enforces the constraint), for example the viscosity, the normal stress differences or the relaxation time being functions of the pressure. As considerable evidence exists for the viscosity being a function of the pressure in many organic liquids that are essentially incompressible (see 2] for the literature before 1931 and the recent paper by Bulicek et al. 3. where references are provided for more recent experimental papers), one expects the relaxation time of geological materials such as the earth's mantle to depend on the pressure. In fact, it has been experimentally verified that the viscosity can depend exponentially on the pressure (see the recent experiment of Bair and Kottke [1, while the density of the fluid increases by a few percent (see Dowson and Higginson [8], Rajagopal [19]). The viscosity increases by as much as 30 percent in many organic liquids. While none of the classical fluid models or simple fluid models can handle this dependence of viscosity on pressure, implicit constitutive models for fluids can describe such dependence (see Rajagopal [18 and Rajagopal 19] for a detailed discussion of implicit models for fluids) 3 . Nor can the classical Bingham fluid model (see [15]) or the Herschel-Bulkley [13] fluid model be cast within the classical framework. They can however be formulated as the symmetric part of the velocity gradient depending on the stress. Recently, Malek et al. 14 have studied the flows of stress power-law fluids, namely fluids wherein one can express the symmetric part of the velocity gradient as a power-law function of the stress. They find that such fluids exhibit a markedly different response from that of the classical power-law fluid in flows between infinite parallel plates and between cylindrical annulis. We are interested in considering a different subclass of implicit fluid models, but before describing them we would like to motivate them by looking at the solid mechanical analogs that have been reasonably well studied, and discussing them will provide guidance to understanding the fluid problem and also to understanding some fundamental differences between such relationships. The solid mechanics counterpart of implicit fluid models between the Cauchy stress and the symmetric part of the velocity gradient are implicit models for solids, with the relation being between the Cauchy stress and the Cauchy Green stretch tensor (see Rajagopal [19, Rajagopal [20, Rajagopal and Srinivasa [21]). It is well known that there are many solids, especially rubber-like materials, that exhibit limiting chain extensibility. A simple model that takes limiting chain extensibility into account is the model due to Gent (see 22]) wherein the stored energy includes a parameter that is referred to as the limiting chain parameter. We are not interested in a detailed discussion of the limiting chain

\footnotetext{
${ }^{2}$ According to Truesdell and Noll 24 the Cauchy stress in a generalized simple fluid depends on the history of the deformation gradient of the fluid. If the fluid is compressible, it will also depend on the density of the fluid.

${ }^{3}$ The idea of implicit models is not new as such models have been used to describe viscoelastic fluids (e.g. Maxwell fluid, Oldroyd-B fluid, Burgers fluid, etc.). Interestingly, in such rate-type fluid models, one can once again have the material moduli, such as the relaxation time, depending on the pressure.
} 
extensibility models in elasticity. What is of consequence is the fact that these models form a subclass of elastic solid models that are implicit. Rajagopal and Saccomandi 22 have discussed the status of models with limiting chain extensibility within the context of implicit constitutive equations for solids and they have also considered special solutions within the context of such models. More recently, Rajagopal 23 has studied several problems within the context of nonlinear elastic solids that exhibit limiting strain.

It is the appropriate counterparts of such solid models which exhibit limiting chain extensibility within the context of fluid mechanics that we are interested in. Within the context of fluid theories, we are interested in the class of fluid models with limiting shear rate (shear strain) when subject to shear stresses. It turns out, just as in the case of solid bodies, that the class of fluid bodies that exhibit limiting shear rate can be studied within the context of implicit fluid models (see Rajagopal 19] and Rajagopal [18, for a detailed discussion of implicit fluid theories). This paper is devoted to the study of such fluid models with limiting shear rate. However, before we discuss the special initial-boundary value problem that we are interested in, it is worthwhile to discuss briefly the results that have been found in the case of solids with limiting stretch so that we can compare and contrast the results that are obtained in the course of this study. In fact, there are some striking differences with regard to the results. Recently, Farina et al. [10], [11] studied the one-dimensional problem for an elastic solid with limited stretch and proved that the equations governing the deformations of such a body have a unique solution. However, they showed that the problem obtained from a limiting procedure for a constitutive model with a piecewise linear elastic response showed a very interesting feature, namely that the limit does not lead to the solution of the model with a threshold. On the other hand, in the case of a fluid model we can show that the limit of the procedure with a piecewise linear response does lead to the limiting strain rate (see Section 5.11). As we shall see this has to do with the dissipative structure of the interface.

In this paper we study the response of the counterpart in fluid mechanics of the above model, namely those which exhibited a limited shear rate response (see Figure 1). Below a certain threshold for the shear rate, the fluid behaves like a linearly viscous NavierStokes fluid. However, once a threshold in the shear rate is reached, the shear rate of the fluid remains constant even when the shear stress is increased. Such a behavior can be viewed as the opposite of a Bingham fluid-like response wherein the shear rate is zero until a critical value in the shear stress is reached, beyond which the fluid flows like a linearly viscous fluid.

The paper is organized as follows. In the next section we introduce the general representation for implicit constitutive models between the Cauchy stress and the symmetric part of the velocity gradient, and the special implicit constitutive model that we shall consider in this paper. In section 3 we consider special unsteady unidirectional flows between two infinite parallel plates which leads to two distinct regimes of flow for the fluid model under consideration and prove a uniqueness theorem for the flow. In section 4 we consider the gravity-driven flow of the implicit fluid in a vertical cylinder. In the following section we discuss how to measure the threshold value for the stress below which it behaves like a Navier-Stokes fluid and the viscosity and also consider the flow of a shear-thickening fluid. We end the paper with a few concluding remarks. 
2. The general model. A general class of implicit constitutive relations for an incompressible fluid can be defined through (see [18, [19])

$$
g(\mathbf{T}, \mathbf{D})=0,
$$

where $\mathbf{T}$ is the Cauchy stress and $\mathbf{D}$ is the symmetric part of the velocity gradient, subject to the requirement

$$
\operatorname{tr} \mathbf{D}=0 .
$$

If $\mathbf{g}$ is an isotropic function, then it has the following representation [17]:

$$
\begin{aligned}
& \alpha_{o} \mathbf{I}+\alpha_{1} \mathbf{T}+\alpha_{2} \mathbf{D}+\alpha_{3} \mathbf{T}^{2}+\alpha_{4}^{2} \mathbf{D}^{2}+\alpha_{5}(\mathbf{T D}+\mathbf{D T}) \\
& +\alpha_{6}\left(\mathbf{T}^{2} \mathbf{D}+\mathbf{D} \mathbf{T}^{2}\right)+\alpha_{7}\left(\mathbf{D}^{2} \mathbf{T}+\mathbf{T D}^{2}\right)+\alpha_{8}\left(\mathbf{T}^{2} \mathbf{D}^{2}+\mathbf{D}^{2} \mathbf{T}^{2}\right)=0,
\end{aligned}
$$

where $\alpha_{i}, i=0,1, \ldots, 8$, depend at most upon $\operatorname{tr} \mathbf{T}, \operatorname{tr} \mathbf{T}^{2}, \operatorname{tr} \mathbf{D}^{2}, \operatorname{tr} \mathbf{T}^{3}, \operatorname{tr} \mathbf{D}^{3}, \operatorname{tr}(\mathbf{T D})$, $\operatorname{tr}\left(\mathbf{T}^{2} \mathbf{D}\right), \operatorname{tr}\left(\mathbf{D}^{2} \mathbf{T}\right)$ and $\operatorname{tr}\left(\mathbf{T}^{2} \mathbf{D}^{2}\right)$ as well as on other parameters characterizing the material (e.g., the mass density $\rho$ ). If the fluid were compressible, or if other variables such as the temperature were to be taken into account, in that they appear in the function $g$, then the material moduli would also depend on the density and temperature.

Within this framework we consider the following implicit constitutive relation:

$$
\beta_{o} \mathbf{I}+\beta_{1}(\widehat{\mathbf{T}}-2 \eta \mathbf{D})=0 \text { with }\left\{\begin{array}{l}
\beta_{o}=\left[I I_{\mathbf{D}}^{1 / 2}-\frac{\sigma_{o}}{2 \eta}\right] \Theta\left(I I_{\widehat{\mathbf{T}}}^{1 / 2}-\sigma_{o}\right), \\
\beta_{1}=\Theta\left(\sigma_{o}-I I_{\widehat{\mathbf{T}}}^{1 / 2}\right),
\end{array}\right.
$$

where:

- $\widehat{\mathbf{T}}$ is the deviatoric part of $\mathbf{T}$, defined as follows:

$$
\widehat{\mathbf{T}}=\mathbf{T}-(1 / 3 \operatorname{tr} \mathbf{T}) \mathbf{I} .
$$

We recall that such a decomposition has a purely geometrical character without any appeal to physical principles.

- $I I_{\widehat{\mathbf{T}}}$ and $I I_{\mathbf{D}}$ are the second invariant of $\widehat{\mathbf{T}}$ and of $\mathbf{D}$, respectively, i.e. $I I_{\widehat{\mathbf{T}}}=$ $1 / 2 \operatorname{tr} \widehat{\mathbf{T}}^{2}$ and $I I_{\mathbf{D}}=1 / 2 \operatorname{tr} \mathbf{D}^{2}$.

- $\Theta$ is the Heaviside function

$$
\Theta(z)= \begin{cases}0, & \text { if } z<0, \\ 1, & \text { if } z \geqslant 0 .\end{cases}
$$

- $\sigma_{o},\left[\sigma_{o}\right]=P a$, plays the role of a threshold stress.

- $\eta,[\eta]=P a s$, is the fluid viscosity. Both $\sigma_{o}$ and $\eta$ are constant and positive.

In particular, when condition $I I_{\widehat{\mathrm{T}}}^{1 / 2} \leq \sigma_{o}$ is fulfilled, we have the usual law for viscous fluids:

$$
\widehat{\mathbf{T}}=2 \eta \mathbf{D} \Rightarrow \mathbf{T}=-p \mathbf{I}+2 \eta \mathbf{D},
$$

where

$$
p=-\frac{1}{3} \operatorname{tr} \mathbf{T}
$$

\footnotetext{
${ }^{4} \operatorname{tr} \mathbf{A}$ denotes the trace of the tensor $\mathbf{A}$.
} 
When $I I_{\widehat{\mathbf{T}}}^{1 / 2}>\sigma_{o}, I I_{\mathbf{D}}^{1 / 2}$ becomes constant and equals the value corresponding to $I I_{\widehat{\mathbf{T}}}^{1 / 2}$ at the threshold, i.e. $I I_{\mathbf{D}}^{1 / 2}=\frac{\sigma_{o}}{2 \eta}$. Thus, if the stress exceeds a certain threshold, it becomes undetermined while $\mathbf{D}$ stays on a manifold defined by $2 \eta^{2} \operatorname{tr} \mathbf{D}^{2}=\sigma_{o}^{2}$.

We consider the case in which the spatial domain occupied by the material at time $t$ is divided by a sharp interface $\mathcal{S}$ into two subdomains, characterized by $I I_{\widehat{\mathbf{T}}}$ being above or below the threshold. Thus, at the interface, the transition between two regimes takes place.

3. Flow between parallel planes. We start by considering the one-dimensional motion of a fluid within a layer confined between two infinite parallel plates at $y= \pm h$. Let the $x$-axis be the direction of the flow:

$$
\vec{v}(y, t)=v(y, t) \vec{e}_{x},
$$

so that the incompressibility constraint is automatically met. The symmetric part of the velocity gradient is 5

$$
\mathbf{D}=\frac{1}{2}\left(\begin{array}{ccc}
0 & \dot{\gamma} & 0 \\
\dot{\gamma} & 0 & 0 \\
0 & 0 & 0
\end{array}\right) \text {, where } \dot{\gamma}=v_{y}
$$

Hence

Concerning $\mathbf{T}$, we have

$$
I I_{\mathbf{D}}^{1 / 2}=\frac{1}{2}|\dot{\gamma}|
$$

$$
\mathbf{T}=\left(\begin{array}{ccc}
-p & \sigma & 0 \\
\sigma & -p & 0 \\
0 & 0 & -p
\end{array}\right),
$$

because of symmetry considerations. In particular, $I I_{\widehat{\mathbf{T}}}^{1 / 2}=|\sigma|$. Thus, we may discriminate between the two regimes as follows:

$$
\begin{aligned}
& |\sigma| \leq \sigma_{o} \quad \Leftrightarrow \quad \sigma=\eta \dot{\gamma}, \\
& |\sigma|>\sigma_{o} \quad \Leftrightarrow \quad|\dot{\gamma}|=\dot{\gamma}_{o}=\frac{\sigma_{o}}{\eta} .
\end{aligned}
$$

The implicit constitutive law (2.1) then leads to

$$
g(\sigma, \dot{\gamma})=(\sigma-\eta \dot{\gamma}) \Theta\left(\sigma_{o}-\sigma\right)+\Theta\left(\sigma-\sigma_{o}\right)\left(\dot{\gamma}-\dot{\gamma}_{o}\right)=0,
$$

and it is shown in Figure 1 ,

We remark that strain rate vs. stress is a function whereas stress vs. strain rate is a graph. The implicit law (3.1) shows that when $\sigma<\sigma_{o}$ the material behaves like a viscous Newtonian fluid with viscosity $\eta$, whereas for $\sigma \geqslant \sigma_{o}$ the strain rate (or shear rate) is fixed, $\dot{\gamma}=\dot{\gamma}_{o}$.

The implicit law (3.1) parallels the one presented in [10] where we studied the onedimensional problem for an elastic material with a limited extensibility range.

\footnotetext{
${ }^{5} v_{y}$ and $v_{t}$ stand for $\frac{\partial v}{\partial y}$ and for $\frac{\partial v}{\partial t}$, respectively.
} 

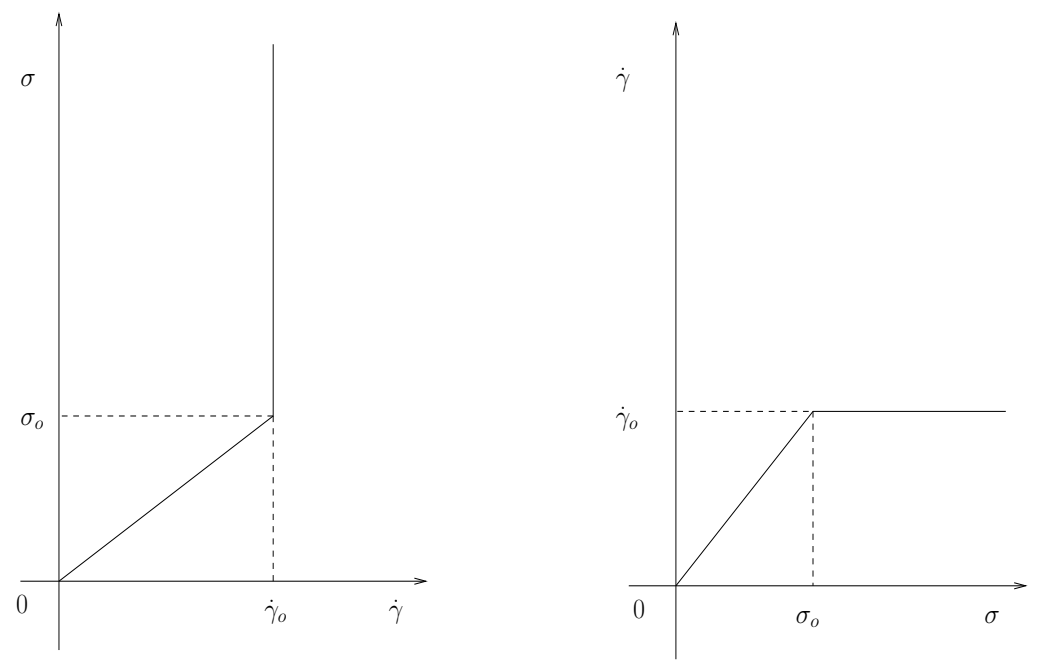

Fig. 1. Constitutive relation linking the shear rate $\dot{\gamma}$ with the shear stress $\sigma$.

Due to symmetry reasons, we may confine our attention to the region $0 \leq y \leq h$. The interface $\mathcal{S}$ has the equation $y=s(t)$ and is not a material surface. In particular, for $0 \leq y \leq s(t)$, the stress is below threshold (see Figure 2).

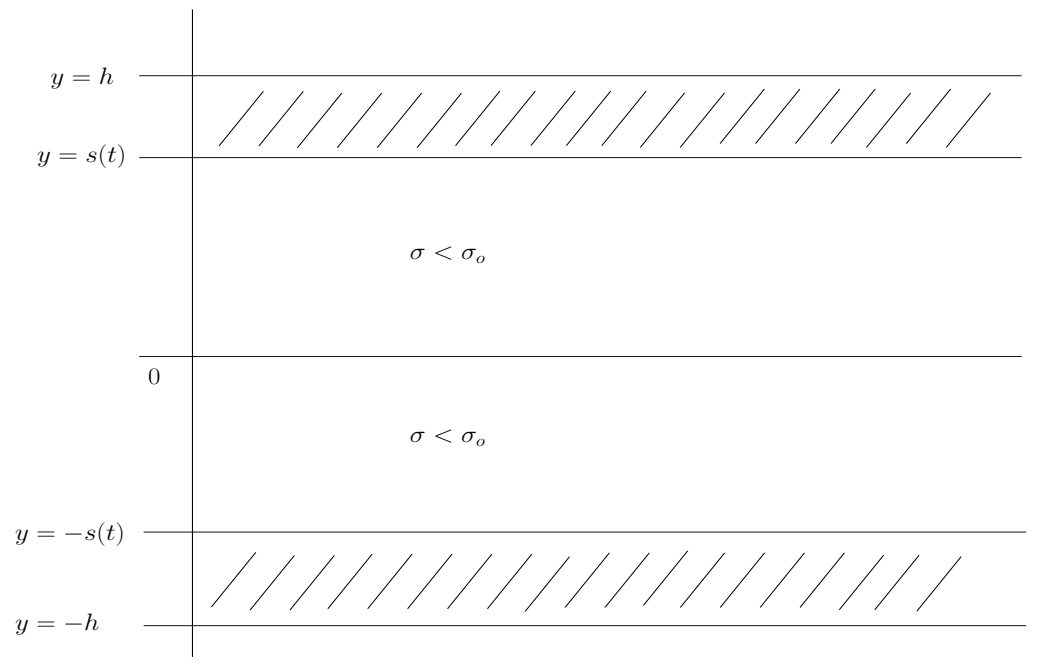

Fig. 2. Flow between two parallel planes. The interface $y=$ $s(t)$ is the free boundary separating the inner core (where the stress is below the threshold) and the region where the stress exceeds the threshold (fixed shear rate).

3.1. Mathematical formulation. We consider a flow driven by a known pressure gradient $f_{o}>0$. In particular, we analyze the case in which $f_{o}$ is constant (the problem can be extended to the case of a time-dependent pressure gradient $f(t)$ ). In the region 
$s(t) \leqslant y \leqslant h$ we have

$$
v_{y}(y)=-\frac{\sigma_{o}}{\eta} \Rightarrow v(y)=\frac{\sigma_{o}}{\eta}(h-y),
$$

since the no-slip condition is imposed on $y=h$. Hence

$$
v\left(s^{+}\right)=\frac{\sigma_{o}}{\eta}(h-s) .
$$

Relations (3.2) express the fact that $\dot{\gamma}$ is fixed in the region where $\sigma \geqslant \sigma_{o}$. Consequently the velocity decreases linearly to 0 as $y$ approaches the boundary.

In the domain $0 \leqslant y \leqslant s(t)$ the problem for $v$ and $s$ is

$$
\begin{cases}\rho v_{t}-\eta v_{y y}=f_{o}, & 0<y<s(t), \quad t>0, \\ v(y, 0)=v_{o}(y), & 0<y<s(0), \\ v_{y}(0, t)=0, & t>0, \\ v\left(s^{-}, t\right)=\frac{\sigma_{o}}{\eta}(h-s), & t>0, \\ v_{y}\left(s^{-}, t\right)=-\frac{\sigma_{o}}{\eta}, & t>0, \\ s(0)=b, & 0<b \leqslant h\end{cases}
$$

where:

- $\rho$ is the fluid density (assumed constant).

- $v_{o}(y)$ is the initial velocity, and $b$ is the initial position of the free boundary.

- $v_{y}(0, t)=0$ expresses the symmetry of $v$ across $y=0$.

- $v\left(s^{-}, t\right)=\sigma_{o} / \eta(h-s)$ expresses the assumption

$$
\llbracket v \rrbracket=0,
$$

where

$$
\llbracket(\cdot) \rrbracket=\left.(\cdot)\right|_{y=s^{+}}-\left.(\cdot)\right|_{y=s^{-}}
$$

denotes the jump of the quantity $(\cdot)$ across the interface. We remark that condition (3.4) ensures that slip phenomena do not occur at the interface $y=s(t)$. In particular, (3.4) also ensures the continuity of the stress (see, for instance, 4, Chapter 8).

- $v_{y}(s, t)=-\sigma_{o} / \eta$ expresses the requirement for the stres: 6 to attain the threshold value at the interface $y=s(t)$.

We assume the following compatibility conditions for the initial data:

$$
v_{o}(b)=\frac{\sigma_{o}}{\eta}(h-b), \quad v_{o}^{\prime}(b)=-\frac{\sigma_{o}}{\eta}, \text { and } v_{o}^{\prime}(0)=0 .
$$

We cast problem (3.3) in a nondimensional form by means of the rescaling

$$
y=y^{*} h, \quad t=t^{*} t_{c}, \quad v=v^{*} v_{c}, \quad \sigma=\sigma^{*} \sigma_{o}, \quad v_{o}=v_{o}^{*} v_{c}, \quad s=s^{*} h, \quad b=b^{*} h,
$$

and we set

$$
t_{c}=\frac{\rho h^{2}}{\eta}, \quad v_{c}=\frac{f_{o} h^{2}}{\eta}
$$

\footnotetext{
${ }^{6}$ Recall that in the region $0<y<s(t), \sigma(y, t)=\eta v_{y}(y, t)$.
} 
as the characteristic time and velocity, respectively. Problem (3.3) becomes (we omit "*" to simplify the notation)

$$
\left\{\begin{array}{l}
v_{t}-v_{y y}=1, \quad 0<y<s(t), \quad t>0, \\
v(y, 0)=v_{o}(y), \quad 0<y<s(0), \\
v_{y}(0, t)=0, \quad t>0, \\
v(s, t)=\lambda(1-s), \quad t>0, \\
v_{y}(s, t)=-\lambda, \quad t>0 \\
s(0)=b, \quad 0<b \leqslant 1
\end{array}\right.
$$

where

$$
\lambda=\frac{\sigma_{o}}{f_{o} h}>0 .
$$

The compatibility conditions (3.5) become $v_{o}(b)=\lambda(1-b), v_{o}^{\prime}(0)=0$ and $v_{o}^{\prime}(b)=-\lambda$. We observe that problem (3.6) has Cauchy conditions on the free boundary which make it different from the parabolic free boundary problem describing Bingham flows (see [5]).

3.1.1. Auxiliary problems. To study problem (3.6) we follow a usual technique (see e.g. [5, 9], 16]) introducing the transformation

$$
z(y, t)=v_{y}(y, t)
$$

whose inverse is given by

$$
v(y, t)=\lambda(1-s)-\int_{y}^{s} z(\xi, t) d \xi
$$

The problem for $z(y, t)$ becomes

$$
\left\{\begin{array}{l}
z_{t}-z_{y y}=0, \quad 0<y<s(t), \quad t>0, \\
z(y, 0)=z_{0}(y), \quad 0<y<s(0), \\
z(0, t)=0, \quad t>0, \\
z(s, t)=-\lambda, \quad t>0, \\
z_{y}(s, t)=-1, \quad t>0 \\
s(0)=b, \quad 0<b \leqslant 1
\end{array}\right.
$$

where $z_{o}(y)=v_{o}^{\prime}(y)$. We notice that (3.9) is still of Cauchy type. We also consider the transformation

$$
w(y, t)=v_{t}(y, t)
$$


obtaining the following free boundary problem of Stefan type:

$$
\left\{\begin{array}{l}
w_{t}-w_{y y}=0, \quad 0<y<s(t), \quad t>0 \\
w(y, 0)=w_{o}(y), \quad 0<y<s(0) \\
w_{y}(0, t)=0, \quad t>0 \\
w(s, t)=0, \quad t>0 \\
w_{y}(s, t)=\dot{s}, \quad t>0 \\
s(0)=b, \quad 0<b \leqslant 1
\end{array}\right.
$$

where $w_{o}(y)=v_{o}^{\prime \prime}(y)+1$. It is easy to see that

$$
z(y, t)=s-y-\lambda-\int_{y}^{s} w(\xi, t) d \xi,
$$

so that the solution of problem (3.6) can be obtained from the solution of (3.10) by means of (3.11) and (3.8). The free boundary condition (3.10) 5 can be rewritten in the following integral form:

$$
s(t)=\lambda+\int_{0}^{s(t)} w(y, t) d y,
$$

exploiting Green's theorem applied to the equation (3.10) 1 .

We assume the following hypotheses on the data:

(H1) $v_{o}(y) \in C^{3}[0, b]$, and $v_{o}(b)=\lambda(1-b), 0<b \leq 1$.

(H2) $-\lambda \leqslant v_{o}^{\prime}(y) \leqslant 0$ in $[0, b]$ and $v_{o}^{\prime}(b)=-\lambda, v_{o}^{\prime}(0)=0$.

(H3) $v_{o}^{\prime \prime}(y)+1 \geqslant 0$ in $[0, b], v_{o}^{\prime \prime}(b)=-1$.

(H4) $f_{o} h \geq \sigma_{o}$.

Hypothesis (H3) can be replaced by the following:

$\left(\mathrm{H} 3^{*}\right) v_{o}^{\prime \prime}(y)+1 \leqslant 0$ in $[0, b]$, and $v_{o}^{\prime \prime}(b)=-1$.

As we shall see later, hypothesis (H3) produces a decreasing $y=s(t)$, while $\left(\mathrm{H} 3^{*}\right)$ an increasing $y=s(t)$.

Definition 1. Let us define

$$
D_{s, T}=\left\{(y, t) \in \mathbb{R}^{2}: 0<y<s(t), 0<t<T\right\} .
$$

A pair $(w(y, t), s(t))$ is called a solution of Problem (3.10) in the time interval $[0, T]$, with $T>0$ if

1. $s(t) \in C^{1}[0, T]$ and $s(t)$ is positive.

2. $w(y, t) \in C^{2,1}\left(D_{s, T}\right) \cap C^{1,0}\left(\overline{D_{s, T}}\right)$.

3. $w(y, t)$ and $s(t)$ satisfy all the equations of Problem (3.10).

\subsubsection{Analytical results.}

Theorem 1. Under hypotheses (H1)-(H4), problem (3.10) admits a unique solution $(w, s)$ for any time $[0, T]$ in the sense of Definition 1, with $\dot{s}(t)<0$. When (H3) is replaced by $\left(\mathrm{H} 3^{*}\right)$, then $\dot{s}(t)>0$. 
Proof. Global existence and uniqueness of a solution can be proved following the results of [16], 9]. The maximum principle and Hopf's lemma (see [12]) ensure the monotonicity of the free boundary, i.e. $\dot{s}(t)<0\left(\dot{s}(t)>0\right.$ if $\left(\mathrm{H} 3^{*}\right)$ holds). Indeed $w(y, t)$ takes its minimum along the whole boundary $y=s(t)$, so that $w_{y}(s, t)=\dot{s}<0$ $\left(w_{y}(s, t)=\dot{s}<0\right.$ under $\left(\mathrm{H} 3^{*}\right)$, by Hopf's lemma).

Remark 1. From the hypotheses (H1)-(H4) we have that $7<b$. Indeed

$$
v_{o}^{\prime \prime}(y) \geqslant-1 \quad \Longrightarrow \int_{0}^{b} v_{o}^{\prime \prime}(y) d y>-b \quad \Longrightarrow \lambda<b .
$$

Moreover

$$
\lambda<s(t) \leqslant b, \quad \forall t>0 .
$$

When (H3) is replaced by $\left(\mathrm{H} 3^{*}\right)$,

$$
v_{o}^{\prime \prime}(y) \leqslant-1 \quad \Longrightarrow \int_{0}^{b} v_{o}^{\prime \prime}(y) d y<-b \quad \Longrightarrow \lambda>b,
$$

and

$$
b \leqslant s(t)<\lambda, \quad \forall t>0 .
$$

Once $(w, s)$ is determined, the solution of problem (3.9) is obtained by means of (3.11), whereas the solution of problem (3.6) is obtained by means of (3.8). Some estimates for $v$ and its derivatives are given in the following.

Lemma 1. Under hypotheses (H1)-(H4), the solution $v(y, t)$ of problem (3.6) is such that:

1. $-\lambda \leqslant v_{y}(y, t) \leqslant 0$.

2. $0 \leqslant v_{t}(y, t) \leqslant \max _{[0, b]}\left[v_{o}{ }^{\prime \prime}(y)+1\right]$.

3. $0 \leqslant v(y, t) \leqslant \lambda$.

When (H3) is replaced by $\left(\mathrm{H} 3^{*}\right)$, result 2 must be replaced by

$2^{*} \cdot \min _{[0, b]}\left[v_{o}^{\prime \prime}+1\right] \leqslant v_{t}(y, t) \leqslant 0$.

Proof. Results 1, 2 and $2^{*}$ follow from the weak version of the maximum principle applied to problems (3.9) and (3.10), respectively. Point 3 follows from (3.8) and $-\lambda \leqslant$ $z \leqslant 0$.

REMARK 2. Problem (3.6) admits the stationary solution

$$
v_{\infty}(y)=-\frac{y^{2}}{2}+\left(s_{\infty}-\frac{s_{\infty}^{2}}{2}\right),
$$

with

$$
s_{\infty}=\frac{\sigma_{o}}{f_{o} h}=\lambda .
$$

Of course the stationary free boundary $s_{\infty}$ has a physical meaning only if $s_{\infty} \leqslant 1$, that is,

$$
f_{o} \geqslant \frac{\sigma_{o}}{h},
$$

which is guaranteed by hypothesis (H4).

\footnotetext{
${ }^{7}$ Recall that $b \leqslant 1$ from (H1).
} 
Lemma 2. The solution $(v, s)$ of problem (3.6) tends asymptotically to $\left(v_{\infty}, s_{\infty}\right)$ for $t \rightarrow \infty$.

Proof. Consider problem (3.9) . Equation (3.9) 1 can be rewritten as $(y z)_{t}-\left(y z_{y}-z\right)_{y}=$ 0 . Integrating over $D_{s, t}$ and applying Green's formula we get

$$
\oint_{\partial D_{s, t}}(y z) d y+\left(y z_{y}-z\right) d \tau=0 .
$$

Therefore

$$
\int_{0}^{t}\left(s-s_{\infty}\right) d \tau=\frac{\lambda}{2}\left(b^{2}-s^{2}\right)+\int_{0}^{b} y z_{o}(y) d y-\int_{0}^{s} y z(y, t) d y .
$$

The right-hand side of (3.14) is bounded because of Remark 1 1 and condition 1 of Lemma (1) Thus necessarily

$$
\lim _{t \rightarrow \infty} s(t)=s_{\infty}=\lambda
$$

and

$$
\lim _{t \rightarrow \infty} v(y, t)=v_{\infty}(y)=-\frac{y^{2}}{2}+\left(s_{\infty}-\frac{s_{\infty}^{2}}{2}\right)
$$

uniformly in $t$.

3.1.3. Monotone dependence of the solution on the initial data. Let us consider two different sets of data $\left(b_{1}, v_{o 1}\right),\left(b_{2}, v_{o 2}\right)$ satisfying $(\mathrm{H} 1)-(\mathrm{H} 4)$ and such that

$$
0<b_{1}<b_{2} \leqslant 1, \quad v_{o 1}^{\prime \prime}(y) \leqslant v_{o 2}^{\prime \prime}(y), \quad y \in\left[0, b_{1}\right] .
$$

REMARK 3. Integrating the second inequality of (3.15) between 0 and $y$ we get

$$
v_{o 1}^{\prime}(y) \leqslant v_{o 2}^{\prime}(y)
$$

Recalling then that

$$
v_{i o}(y)=\lambda\left(1-b_{i}\right)-\int_{y}^{b_{i}} v_{o i}^{\prime}(y) d y
$$

for $i=1,2$, we get

$$
v_{o 2}(y)-v_{o 1}(y)=\lambda\left(b_{1}-b_{2}\right)+\int_{y}^{b_{1}}\left(v_{o 1}^{\prime}(\xi)-v_{o 2}^{\prime}(\xi)\right) d \xi-\int_{b_{1}}^{b_{2}} v_{o 2}^{\prime}(\xi) d \xi .
$$

From hypothesis (H2),

$$
-\int_{b_{1}}^{b_{2}} v_{o 2}^{\prime}(\xi) d \xi \leqslant \lambda\left(b_{2}-b_{1}\right)
$$

Thus

$$
v_{o 2}(y)-v_{o 1}(y) \leqslant \int_{y}^{b_{1}}\left(v_{o 1}^{\prime}(\xi)-v_{o 2}^{\prime}(\xi)\right) d \xi \leqslant 0,
$$

where the last inequality is by virtue of (3.16). Therefore

$$
v_{o 1}^{\prime \prime} \leqslant v_{o 2}^{\prime \prime} \Longrightarrow v_{o 1}^{\prime} \leqslant v_{o 2}^{\prime} \Longrightarrow v_{o 1} \geqslant v_{o 2} \text {. }
$$


Let us consider the solutions $\left(w_{1}, s_{1}\right),\left(w_{2}, s_{2}\right)$ corresponding to the set of data given in (3.15) and define

$$
U(y, t)=w_{2}(y, t)-w_{1}(y, t) .
$$

Since $s_{1}, s_{2}$ are continuously differentiable and $b_{1}<b_{2}, s_{1}(t)<s_{2}(t)$ for sufficiently small times. Let us suppose that there exists a time $\bar{t} \in(0, T]$ such that $s_{1}(\bar{t})=s_{2}(\bar{t})$. Since $\dot{s}_{1}, \dot{s}_{2}<0$ we have that

$$
\dot{s}_{1}(\bar{t}) \leqslant \dot{s}_{2}(\bar{t}) .
$$

In $D_{s_{1}, \bar{t}}$ the function $U(y, t)$ solves

$$
\left\{\begin{array}{l}
U_{t}-U_{y y}=0, \quad 0<y<s_{1}(t), \quad 0<t<\bar{t}, \\
U(y, 0)=v_{o 2}^{\prime \prime}(y)-v_{o 1}^{\prime \prime}(y) \geqslant 0, \quad 0<y<b_{1}, \\
U_{y}(0, t)=0, \quad 0<t<\bar{t}, \\
U\left(s_{1}, t\right)=w_{2}\left(s_{1}, t\right) \geqslant 0, \quad 0<t<\bar{t} \\
U_{y}\left(s_{1}, t\right)=w_{2 y}\left(s_{1}, t\right)-\dot{s}_{1}, \quad 0<t<\bar{t} .
\end{array}\right.
$$

Let $\bar{y}=s_{1}(\bar{t})=s_{2}(\bar{t})$. By the maximum principle, $U(y, t) \geqslant 0$ in $D_{s_{1}, \bar{t}}$ and $U(\bar{y}, \bar{t})=0$; that is, $(\bar{y}, \bar{t})$ is a minimum. By Hopf's lemma, $U_{y}(\bar{y}, \bar{t})<0$, contradicting $(3.18)_{5}$ where $U_{y}(\bar{y}, \bar{t})=\dot{s}_{2}(\bar{t})-\dot{s}_{1}(\bar{t}) \geqslant 0$, because of (3.17). Thus we have proved the following.

Lemma 3. Under hypotheses (H1)-(H4), the solutions $\left(v_{1}, s_{1}\right),\left(v_{2}, s_{2}\right)$ relative to the set of data $0<b_{1}<b_{2} \leqslant 1, v_{o 1}^{\prime \prime}(y) \leqslant v_{o 2}^{\prime \prime}(y), y \in\left[0, b_{1}\right]$ are such that

1. $v_{2 t}(y, t) \geqslant v_{1 t}(y, t), \quad(y, t) \in D_{s_{1}, T}$,

2. $v_{2 y}(y, t) \geqslant v_{1 y}(y, t), \quad(y, t) \in D_{s_{1}, T}$,

3. $v_{2}(y, t) \leqslant v_{1}(y, t), \quad(y, t) \in D_{s_{1}, T}$,

4. $s_{1}(t)<s_{2}(t)$ for all $T>0$.

Proof. Result 1 has already been proved. Result 2 can be easily obtained. Indeed, from 1 , we have

$$
\int_{0}^{y} v_{2 t}(y, t) d y \geqslant \int_{0}^{y} v_{1 t}(y, t) d y \Longrightarrow \int_{0}^{y} v_{2 y y}(y, t) d y \geqslant \int_{0}^{y} v_{1 y y}(y, t) d y,
$$

which, once integrated, gives 2 . Result 3 is obtained from (3.8). Indeed

$$
v_{i}=\lambda\left(1-s_{i}\right)-\int_{y}^{s_{i}} z_{i}(\xi, t) d \xi, \quad i=1,2 .
$$

Thus

$$
v_{2}(y, t)-v_{1}(y, t)=\lambda\left(s_{1}-s_{2}\right)+\int_{y}^{s_{1}}\left(z_{1}(\xi, t)-z_{2}(\xi, t)\right) d \xi-\int_{s_{1}}^{s_{2}} z_{2}(\xi, t) d \xi,
$$

with $y \in\left[0, s_{1}\right], t>0$. From point 1 of Lemma 1]

$$
-\int_{s_{1}}^{s_{2}} z_{2}(\xi, t) d \xi \leqslant \lambda\left(s_{2}-s_{1}\right) .
$$


Thus (3.19) becomes

$$
v_{2}(y, t)-v_{1}(y, t) \leqslant \int_{y}^{s_{1}}\left(z_{1}(\xi, t)-z_{2}(\xi, t)\right) d \xi \leqslant 0,
$$

where the last inequality is due to 2 .

4. Gravity-driven flow in a vertical cylinder. In this section we study the flow in a vertical cylinder of radius $R$. Let $\vec{e}_{z}, \vec{e}_{r}$ be the axia 8 and radial unit normal vectors and let $\vec{v}(r, t)=v(r, t) \vec{e}_{z}$ be the velocity in the domain $0<r<s(t)$, i.e. the region where the strain rate increases linearly with the stress $\sigma$. Analogous to the previous case, in the region of fixed strain rate $s(t)<r<R$, we have

$$
\begin{gathered}
v(r, t)=\frac{\sigma_{o}}{\eta}(R-r), \quad v(s, t)=\frac{\sigma_{o}}{\eta}(R-s), \\
\frac{\partial v}{\partial r}(r, t)=-\frac{\sigma_{o}}{\eta} .
\end{gathered}
$$

Assuming that the flow is driven by gravity and by a constant pressure gradient $f_{o}$ we have

$$
\rho \frac{\partial v}{\partial t}=\rho g+f_{o}+\frac{\eta}{r} \frac{\partial}{\partial r}\left(r \frac{\partial v}{\partial r}\right), \quad 0<r<s(t), \quad t>0 .
$$

This situation corresponds to the flow produced in the vertical pipe when it is connected to a reservoir in which the fluid level is kept constant (or has negligible variations). We add the symmetry condition $v_{r}(0, t)=0$ and the initial condition $v(r, 0)=v_{o}(r)$. Summarizing, the problem for $v$ is the following:

$$
\left\{\begin{array}{l}
\rho \frac{\partial v}{\partial t}=\rho g+f_{o}+\frac{\eta}{r} \frac{\partial}{\partial r}\left(r \frac{\partial v}{\partial r}\right), \quad 0<r<s(t), \quad t>0, \\
v(r, 0)=v_{o}(r), \quad 0<r<s(0), \\
v_{r}(0, t)=0, \quad t>0, \\
v(s, t)=\frac{\sigma_{o}}{\eta}(R-s), \quad t>0, \\
v_{r}(s, t)=-\frac{\sigma_{o}}{\eta}, \quad t>0, \\
s(0)=b, \quad 0<b \leqslant R .
\end{array}\right.
$$

From the rescaling

$$
r=r^{*} R, \quad t=t^{*} t_{c}, \quad v=v^{*} v_{c}, \quad \sigma=\sigma^{*} \sigma_{o}, \quad v_{o}=v_{o}^{*} v_{c}, \quad s=s^{*} R, \quad b=b^{*} R,
$$

where we set

$$
t_{c}=\frac{\rho R^{2}}{\eta}, \quad v_{c}=\frac{\left(\rho g+f_{o}\right) R^{2}}{\eta},
$$

\footnotetext{
${ }^{8} \vec{e}_{z}$ is pointing downward.
} 
we obtain (omitting the "**)

$$
\left\{\begin{array}{l}
\frac{\partial v}{\partial t}=1+\frac{1}{r} \frac{\partial}{\partial r}\left(r \frac{\partial v}{\partial r}\right), \quad 0<r<s(t), \quad t>0 \\
v(r, 0)=v_{o}(r), \quad 0<r<s(0), \\
v_{r}(0, t)=0, \quad t>0, \\
v(s, t)=\lambda(1-s), \quad t>0, \\
v_{r}(s, t)=-\lambda, \quad t>0, \\
s(0)=b, \quad 0<b \leqslant 1,
\end{array}\right.
$$

where now

$$
\lambda=\frac{\sigma_{o}}{\left(\rho g+f_{o}\right) R} .
$$

Analogous to the previous case, we cast the problems for $z=v_{r}$ and $w=v_{t}$, thereby obtaining

$$
\begin{aligned}
& \left\{\begin{array}{l}
z_{t}=z_{r r}+\frac{z_{r}}{r}-\frac{z}{r^{2}}, \quad 0<r<s(t), \quad t>0 \\
z(r, 0)=z_{o}(r), \quad 0<r<s(0), \\
z(0, t)=0, \quad t>0 \\
z(s, t)=-\lambda, \quad t>0 \\
z_{r}(s, t)=\frac{\lambda}{s}-1, \quad t>0 \\
s(0)=b, \quad 0<b \leqslant 1
\end{array}\right. \\
& \left\{\begin{array}{l}
w_{t}=w_{r r}+\frac{w_{r}}{r}, \quad 0<r<s(t), \quad t>0 \\
w(r, 0)=w_{o}(r), \quad 0<r<s(0) \\
w_{r}(0, t)=0, \quad t>0 \\
w(s, t)=0, \quad t>0 \\
w_{r}(s, t)=\left(1-\frac{\lambda}{s}\right) \dot{s}, \quad t>0 \\
s(0)=b, \quad 0<b \leqslant 1
\end{array}\right.
\end{aligned}
$$

where $b R=s_{o}, z_{o}(r)=v_{o}^{\prime}(r)$, and $w_{o}(r)=1+r^{-1} v_{o}^{\prime}(r)+v_{o}^{\prime \prime}(r)$.

It is easy to show that problem (4.1) admits the stationary solution

$$
v_{\infty}=-\frac{r^{2}}{4}+\frac{s_{\infty}}{2}\left(1-\frac{s_{\infty}}{2}\right), \text { with } s_{\infty}=2 \lambda .
$$

The free boundary condition (4.2) 5 can be rewritten by means of the relation

$$
\int_{D_{s, t}}\left[\frac{\partial}{\partial r}\left(r \frac{\partial w}{\partial r}\right)-\frac{\partial}{\partial \tau}(r w)\right] d r=\oint_{\partial D_{s, t}}\left(r \frac{\partial w}{\partial r}\right) d \tau+(r w) d y=0
$$


which yields

$$
(s-\lambda)^{2}=(b-\lambda)^{2}-2 \int_{0}^{b} r w_{o}(r) d r+2 \int_{0}^{s(t)} r w(r, t) d r .
$$

The results obtained in section 3 still hold for the radial case here presented. In particular, (3.13) is replaced by

$$
2 \sigma_{o} \leq\left(\rho g+f_{o}\right) R
$$

5. Measuring the parameters $\sigma_{o}$ and $\eta$. Parameter $9 \sigma_{o}$ and $\eta$ can be measured by means of a rotational viscometer (for the analogous subject concerning Bingham fluids, see [6], 7]). Consider the steady flow of a fluid of the type under investigation between two coaxial cylinders. The inner cylinder (radius $R_{i}$ ) rotates with constant angular velocity $\omega$. The outer cylinder (radius $R_{e}$ ) is kept immobile. As long as the stress is everywhere below the threshold, sufficiently far from the upper and lower ends of the cylinder, the velocity field is $\vec{v}=v(r) \vec{e}_{\theta}$, with

$$
v(r)=\left[\left(\frac{R_{e}}{R_{i}}\right)^{2}-1\right]^{-1}\left[\left(\frac{R_{e}}{r}\right)^{2}-1\right] \omega r .
$$

In a rotational flow,

$$
\mathbf{D}=\left(\begin{array}{ccc}
0 & \frac{r}{2} \frac{\partial}{\partial r}\left(\frac{v}{r}\right) & 0 \\
\frac{r}{2} \frac{\partial}{\partial r}\left(\frac{v}{r}\right) & 0 & 0 \\
0 & 0 & 0
\end{array}\right)
$$

so that $I I_{\mathbf{D}}=\left|\frac{r}{2} \frac{\partial}{\partial r}\left(\frac{v}{r}\right)\right|$.

We denote by $M(r)$ the torque acting at the radius $r$, namely

$$
M(r)=2 \pi r^{2} h\left|\tau_{r \theta}(r)\right|,
$$

where $\tau_{r \theta}$ is the tangential stress and $h$ is the height of the cylinder. In case the stress is everywhere below the threshold, i.e.

$$
\left|\tau_{r \theta}(r)\right|<\sigma_{o}, \quad \forall R_{i} \leq r \leq R_{e},
$$

$M$ is given by

since

$$
M=4 \pi h \eta \frac{R_{i}^{2} R_{e}^{2}}{R_{e}^{2}-R_{i}^{2}} \omega
$$

$$
\tau_{r \theta}\left(R_{i}\right)=\eta r \frac{\partial}{\partial r}\left(\frac{v}{r}\right)=-2 \eta \omega \frac{R_{e}^{2} R_{i}^{2}}{R_{e}^{2}-R_{i}^{2}} \frac{1}{r^{2}} .
$$

Relation (5.1) is used to calculate $\eta$, as it occurs for ordinary Newtonian fluids.

The threshold $\sigma_{o}$ is reached at the wall of the rotating cylinder, and the limit torque is

$$
M_{o}=2 \pi R_{i}^{2} h \sigma_{o}
$$

\footnotetext{
${ }^{9}$ Throughout this section the quantities considered are dimensional.
} 
Hence, for $M>M_{o}$, the domain occupied by the material has to be split into two sub-domains:

- $\Omega_{1}=\left\{R_{i}<r<s(t)\right\}$, where the strain rate is constant and equal to $\sigma_{o} / \eta$,

- $\Omega_{2}=\left\{s(t)<r<R_{e}\right\}$, where the strain rate is smaller than $\sigma_{o} / \eta$.

In $\Omega_{1}$ the strain rate is constant; hence

$$
r \frac{\partial}{\partial r}\left(\frac{v}{r}\right)=-\frac{\hat{\sigma}_{o}}{\eta}, \quad \text { with } \quad \hat{\sigma}_{o}=2 \sigma_{o}
$$

and

$$
\frac{v}{r}=-\frac{\hat{\sigma}_{o}}{\eta} \ln \left(\frac{r}{R_{i}}\right)+\omega .
$$

In $\Omega_{2}$ the problem for $v(r, t)$ is given by

$$
\left\{\begin{array}{l}
\rho v_{t}=\eta\left(v_{r r}+\frac{v_{r}}{r}-\frac{v}{r^{2}}\right), \quad s(t)<r<R_{e}, \quad t>0, \\
v(r, 0)=v_{o}(r), \quad s(0)<r<R_{e}, \\
v\left(R_{e}, t\right)=0, \quad t>0, \\
\frac{v(s, t)}{s}=-\frac{\hat{\sigma}_{o}}{\eta} \ln \left(\frac{s}{R_{i}}\right)+\omega, \quad t>0, \\
\frac{\partial v(s, t)}{\partial r}=-\frac{\hat{\sigma}_{o}}{\eta}\left[\ln \left(\frac{s}{R_{i}}\right)+1\right]+\omega, \quad t>0, \\
s(0)=s_{o}, \quad R_{i}<s_{o}<R_{e},
\end{array}\right.
$$

where $(5.3)_{5}$ derives from the requirement

$$
\left.r \frac{\partial}{\partial r}\left(\frac{v}{r}\right)\right|_{r=s}=-\frac{\hat{\sigma}_{o}}{\eta} .
$$

Looking for the steady solution $\left(s_{\infty}, v_{\infty}\right)$ of (5.3) we get

$$
s_{\infty}^{2}=R_{e}^{2}\left(1-\frac{2 \eta \omega}{\hat{\sigma}_{o}}\right)+2 R_{e}^{2} \ln \left(\frac{s_{\infty}}{R_{i}}\right)
$$

and

$$
\frac{v_{\infty}(r)}{r}=\frac{\hat{\sigma}_{o}}{2 \eta} s_{\infty}^{2}\left[\frac{1}{r^{2}}-\frac{1}{R_{e}^{2}}\right] .
$$

The steady state free boundary $s_{\infty}$ is defined implicitly by (5.4), from which we determine $s_{\infty}=s_{\infty}\left(\omega, R_{i}, R_{e}, \hat{\sigma}_{o}, \eta\right)$. We thus get

$$
M=2 \pi h \hat{\sigma}_{o} \cdot s_{\infty}^{2}\left(\omega, R_{i}, R_{e}, \hat{\sigma}_{o}, \eta\right)
$$

which provides a relation between $M, \omega$ and $\hat{\sigma}_{o}$. If we differentiate expression (5.4) w.r.t. $\omega$ we get

$$
\frac{\partial s_{\infty}}{\partial \omega}=\frac{\eta R_{e}^{2} s_{\infty}}{\hat{\sigma}_{o}\left(R_{e}^{2}-s_{\infty}^{2}\right)}>0 .
$$

Taking the limit $s_{\infty} \rightarrow R_{i}$ is equivalent to letting $\omega \rightarrow \omega_{o}$ (which is the angular velocity corresponding to the torque $M_{o}$ ). Thus from (5.4),

$$
\omega_{o}=\frac{\sigma_{o}}{2 \eta}\left(1-\frac{R_{i}^{2}}{R_{e}^{2}}\right) \text {. }
$$


Expressions (5.1) and (5.6) provide the 1-1 relation between $M$ and $\omega$ below and above the threshold. The torque applied is a function of $\omega$ which is linear up to $\omega_{o}$ and nonlinear for $\omega>\omega_{o}$. Thus, since the viscometer plots $M$ as a function of $\omega$, a simple analysis of this plot provides $\omega_{o}$. Indeed $\omega_{o}$ corresponds to the angular velocity marking the transition to the nonlinear regime. By means of (5.7), we can therefore get the stress threshold $\sigma_{o}$.

5.1. Comparison with a piecewise shear-thickening model. In this section we shall see that the peculiar dynamics described in section 5, can be obtained as a limit case from the piecewise linear viscous model shown in Figure 3 when the slope beyond the threshold tends to infinity.

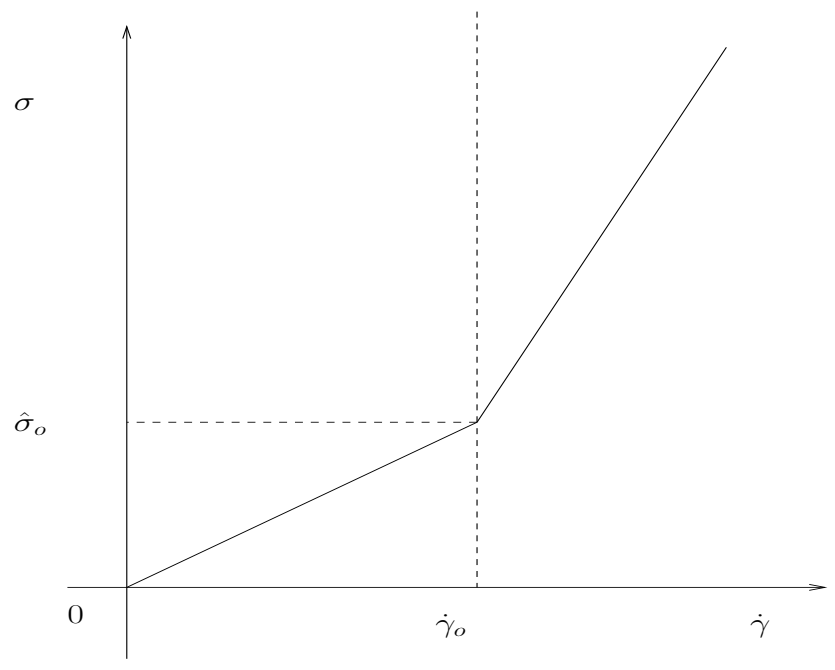

FIG. 3. The piecewise linear viscous model.

Let us consider the following $(\sigma, \dot{\gamma})$ constitutive relation 10 , depending on the parameter $\alpha:$

$$
\sigma=\left\{\begin{array}{ll}
\eta \dot{\gamma}, & 0 \leq \dot{\gamma} \leq \dot{\gamma}_{o}, \\
\alpha \eta\left(\dot{\gamma}-\dot{\gamma}_{o}\right)+\hat{\sigma}_{o}, & \dot{\gamma}_{o}<\dot{\gamma},
\end{array} \quad \text { with } \hat{\sigma}_{o}=\eta \dot{\gamma}_{o},\right.
$$

where the strain rate (or shear rate) is given by $\dot{\gamma}=r \frac{\partial}{\partial r}\left(\frac{v}{r}\right)$. The inverse of (5.8) is

$$
\dot{\gamma}= \begin{cases}\frac{\sigma}{\eta}, & 0 \leq \sigma \leq \hat{\sigma}_{o}, \\ \frac{\sigma-\hat{\sigma}_{o}}{\eta \alpha}+\frac{\hat{\sigma}_{o}}{\eta}, & \sigma>\hat{\sigma}_{o},\end{cases}
$$

so that $\dot{\gamma} \rightarrow \dot{\gamma}_{o}$ if $\alpha \rightarrow+\infty$. In other words, (5.9) tends to the implicit model as $\alpha \rightarrow \infty$.

\footnotetext{
${ }^{10}$ This procedure is nothing but a penalization method.
} 
Considering now the steady problem treated in section [5] for $\omega \geq \omega_{o}$ the domain $\left\{R_{1}<r<R_{e}\right\}$ has to be divided into two subdomains, say $\Omega_{1}$ and $\Omega_{2}$, separated by $r=s$, such that:

- In $\Omega_{1}=\left\{R_{i}<r<s\right\}$ the governing system is

$$
\left\{\begin{array}{l}
r \frac{\partial}{\partial r}\left(\frac{v}{r}\right)=-\frac{\hat{\sigma}_{o}}{\alpha \eta}\left(\frac{s_{\infty}}{r}\right)^{2}-\frac{\hat{\sigma}_{o}}{\eta}\left(1+\frac{1}{\alpha}\right), \\
\frac{v\left(R_{i}\right)}{R_{i}}=\omega .
\end{array}\right.
$$

Hence

$$
\frac{v(r)}{r}-\omega=\frac{s^{2} \hat{\sigma}_{o}}{2 \eta \alpha}\left(\frac{1}{r^{2}}-\frac{1}{R_{i}^{2}}\right)-\frac{\hat{\sigma}_{o}}{\eta}\left(1+\frac{1}{\alpha}\right) \ln \frac{r}{R_{i}} .
$$

- In $\Omega_{2}=\left\{s<r<R_{e}\right\}$ the governing system is

$$
\left\{\begin{array}{l}
r \frac{\partial}{\partial r}\left(\frac{v}{r}\right)=-\frac{\hat{\sigma}_{o}}{\eta} \frac{s^{2}}{r^{2}}, \\
v\left(R_{e}\right)=0
\end{array} \Rightarrow \frac{v(r)}{r}=\frac{s^{2} \hat{\sigma}_{o}}{2 \eta}\left(\frac{1}{r^{2}}-\frac{1}{R_{e}^{2}}\right),\right.
$$

which coincides with (5.5).

- The interface $s$ is determined by solving

$$
\frac{\hat{\sigma}_{o}}{2 \eta}\left(1-\frac{s^{2}}{R_{e}^{2}}\right)=\omega+\frac{\hat{\sigma}_{o}}{2 \eta \alpha}\left(1-\frac{s^{2}}{R_{i}^{2}}\right)-\frac{\hat{\sigma}_{o}}{\eta}\left(1+\frac{1}{\alpha}\right) \ln \frac{s}{R_{i}},
$$

which is a consequence of the no-slip assumption.

Now, when $\alpha \rightarrow \infty$, it is easy to observe that:

(1) The velocity field (5.10) tends to (5.2).

(2) The equation for the interface (5.11) tends to (5.4).

We thus conclude that the shear-thickening model tends to the implicit one depicted in Figure 1, when $\alpha$ tends to infinity. The reason why model (5.9) recovers, in the limit $\alpha \rightarrow+\infty$, the implicit model lies essentially in the fact that the no-slip condition imposed at the interface $r=s$ entails the continuity of the stress and so does not introduce any further dissipation. This is not always the case for materials with constitutive laws of implicit type. For instance, in [10], the presence of an "entropy source" located on the interface makes the model of an elastic material with limited deformability range unattainable by means of the same kind of penalization procedure.

We finally remark that, when $\alpha \rightarrow \infty$, we recover the dynamics associated with the model (5.9) studied in sections 3 and 4 .

6. Conclusions. Working within the framework of implicit constitutive equations, we have developed a new constitutive model which applies to the so-called self-blocking fluids. The peculiarity of the model is that the stress and strain rates are not linked by an invertible relation, the stress being a multi-valued function of the strain rate. In particular when $I I_{\hat{\mathbf{T}}}$ is less than a certain threshold, $\mathbf{T}$ and $\mathbf{D}$ are linked in a linear way, 
but when $I I_{\hat{\mathbf{T}}}$ exceeds such a threshold, $\mathbf{T}$ can attain any value with $\mathbf{D}$ belonging to the manifold $I I_{\mathbf{D}}^{1 / 2}=\sigma_{o} /(2 \eta)$.

We have then analyzed three particular flows: (i) the flow between two parallel planes driven by a given pressure gradient; (ii) the vertical flow in a cylinder driven by gravity; (iii) the axial flow in a rotational viscometer. In each case the mathematical formulation was a free boundary problem of Stefan type, characterized by Cauchy conditions on the free boundary.

\section{REFERENCES}

[1] S. Bair and P. Kottke, Pressure-viscosity relationships for elastohydrodynamics, Tribology Transactions, 46, 289-295, (2003).

[2] P. W. Bridgman, The Physics of High Pressure, New York, MacMillan, 1931.

[3] M. Bulicek, J. Malek and K. R. Rajagopal, Mathematical analysis of unsteady flows of fluids with pressure, shear-rate, and temperature dependent material moduli that slip at solid boundaries, SIAM, 41, 665-707 (2009). MR2515781 (2010f:76021)

[4] J. Coirier, Mécanique des milieux continus, Dunod, Paris, 1997.

[5] E. Comparini, A one-dimesional Bingham flow, J. Math. Anal. Appl., 169, 127-139, (1992). MR $1180677(93 \mathrm{j}: 76008)$

[6] E. Comparini and E. De Angelis, Flow of a Bingham fluid in a concentric cylinder viscosimeter, Adv. Math. Sci. Appl., 6, N. 1, 97-116, (1996). MR1385761(97e:76005)

[7] E. Comparini, Regularization of singular free boundary problems in rotational Bingham flows, Z. Angew. Math. Mech., 77, 543-554, (1997). MR1466444 (98f:76005)

[8] D. Dowson and G. R. Higginson, Elastohydrodynamic Lubrication, the Fundamentals of Roller and Gear Lubrication, Pergamon, Oxford, UK (1996).

[9] A. Fasano and M. Primicerio, General free boundary problems for heat equation, I, J. Math. Anal. Appl., 57, 694-723, 1977. MR0487016 (58:6695a)

[10] A. Farina, A. Fasano, L. Fusi and K.R. Rajagopal, Modelling materials with a stretching threshold, Mathematical Models and Methods in Applied Sciences (M3AS), Vol. 17, Issue 11, (2007), 17991846. MR 2372339(2009g:74013)

[11] A. Farina, A. Fasano, L. Fusi, and K.R. Rajagopal, On the dynamics of an elastic-rigid material, Adv. Math. Sci. Appl. 20 (2010), 193-217. MR2760725

[12] A. Friedman, Partial differential equations of parabolic type, Prentice-Hall, 1964. MR0181836 $(31: 6062)$

[13] W. H. Herschel and R. Bulkley, Konsistenzmessungen von Gummi-Benzollosungen, Colloid Polym. Sci. 39(4), 291-300 (1926).

[14] J. Malek, V. Prusa and K. R. Rajagopal, Generalization of the Navier-Stokes fluid from a new perspective, submitted for publication.

[15] J.G. Oldroyd, A rational formulation of the equations of plastic flow for a Bingham solid, Math. Proc. Camb. Philos. Soc. 43(01), 100-105 (1947). MR0018095(8:240j)

[16] A. Schatz, Free boundary problems of Stephan type with prescribed flux, J. Math. Anal. Appl., 28, 569-580, 1969. MR0267285(42:2187)

[17] A.J.M. Spencer, Theory of invariants, in Continuum Physics, Vol. 3, A.C. Eringen Ed., Academic Press, New York, 1975. MR0468444 (57:8277b)

[18] K.R. Rajagopal, On implicit constitutive theories, Appl. Math., 48 (2003), 279-319. MR1994378 $(2004 \mathrm{j}: 74006)$

[19] K.R. Rajagopal, On implicit constitutive theories for fluids, J. Fluid Mech., 550 (2006), 243-249. MR2263984(2007e:76004)

[20] K.R. Rajagopal, The elasticity of elasticity, Zeitschrift für Angewandte Mathematik und Physik, 58, 309-417 (2007). MR2305717(2008a:74003)

[21] K.R. Rajagopal and A.R. Srinivasa, On the response of non-dissipative solids, Proc. Roy. Soc. London A463, 357-367 (2007). MR2288826 (2007j:74001)

[22] K.R. Rajagopal and G. Saccomandi, The mechanics and mathematics of the effect of pressure on the shear modulus of elastomers, Proc. Roy. Soc. London A 465, 3859-3874 (2009). 
[23] K.R. Rajagopal, Non-linear elastic bodies exhibiting limiting small strain, Mathematics and Mechanics of Solids, 2010, doi:10.1177/1081286509357272.

[24] C. Truesdell and W. Noll, The nonlinear field theories of mechanics, 2nd edn., Springer-Verlag, 1992. MR1215940 (94c:73002)

[25] N.J. Wagner and J.F. Brady, Shear thickening in colloidal dispersion, Physics Today, 62, 27-32 (2009). 